\title{
Microfloral diversity in the lower respiratory tracts of neonates with bacterial infectious pneumonia combined with ventilator-associated pneumonia
}

\author{
WEI LU ${ }^{1-6}$, JIA-LIN YU ${ }^{2-6}$, LU-QUAN LI ${ }^{2-6}$, QI LU ${ }^{2-6}$, ZHENG-LI WANG $^{2-6}$ and YUN PAN ${ }^{2-6}$ \\ ${ }^{1}$ Affiliated Hospital of Zunyi Medical College, Zunyi, Guizhou 563003; ${ }^{2}$ Department of Neonatology; \\ ${ }^{3}$ Pediatrics Research Institution, Children's Hospital of Chongqing Medical University; \\ ${ }^{4}$ Ministry of Education Key Laboratory of Child Development and Disorder; \\ ${ }^{5}$ Key Laboratory of Pediatrics in Chongqing, CSTC2009CA5002; \\ ${ }^{6}$ Chongqing International Science and Technology Cooperation Center for Child Development and Disorders, \\ Chongqing 400014, P.R. China
}

Received July 26, 2015; Accepted August 1, 2016

DOI: $10.3892 / \mathrm{mmr} .2016 .5886$

\begin{abstract}
Bacterial infectious pneumonia is one of the major causes of mortality in neonates, particularly when the neonates suffer from ventilator-associated pneumonia (VAP). However, the causes of pneumonia are difficult to define. Thus, the present study focused on understanding the diversity of microflora in the lower respiratory tract to elucidate the causes. The experimental groups comprised newborns who suffered from infectious pneumonia with or without VAP (IVAP and IP groups, respectively), whereas the control group comprised newborns who suffered from respiratory distress syndrome (RDS) without VAP (RDS group). Following 1, 3 and 5 days of ventilation, sputum samples were collected and the DNA was extracted. The DNA was amplified and separated, and the 16S rDNA was then sequenced and analyzed for diversity. The results of the diversity and Shannon-Wiener indices were ordered as follows: IVAP group < IP group $<$ RDS group. The percentages of Streptococcus sp., Serratia sp. and Achromobacter sp. in the IP and IVAP groups were higher, compared with those in the RDS group, whereas the percentages of Klebsiella sp. and Acinetobacter sp. were lower on day 1 . The percentages of Klebsiella sp. and Streptococcus sp. on days 1 and 3 were ordered as follows: IVAP group > IP group > RDS group, and the percentages of Serratia sp., Acinetobacter sp. and Achromobacter sp. were ordered as follows: IVAP group $<$ IP group $<$ RDS group. After
\end{abstract}

Correspondence to: Dr Jia-Lin Yu, Department of Neonatology, Children's Hospital of Chongqing Medical University, 136, 2 Zhong Shan Street, Yuzhong, Chongqing 400014, P.R. China E-mail: tongxunzuozhe@163.com

Key words: microflora, diversity, neonate, respiratory tract, pneumonia, 16S rDNA, denaturing gradient gel electrophoresis, sequencing
3-5 days, the percentages of Klebsiella sp., Acinetobacter sp., Streptococcus sp., Serratia sp. and Achromobacter sp. in the IVAP group were lower, compared with those in the RDS and IP groups. It was concluded that the decreased microfloral diversity, increased constituent ratios of Klebsiella sp. and Streptococcus sp., and decreased ratios of Serratia sp. and Acinetobacter $\mathrm{sp}$. in the lower respiratory tract of neonates suffering from pneumonia may be indicators of VAP.

\section{Introduction}

Bacterial infectious pneumonia is one of the major causes of mortality in neonates, particularly when they suffer from ventilator-associated pneumonia (VAP). However, it is difficult to detect the pathogens involved in pneumonia (1-3). Thus, it is important to understand the diversity and associations among microflora to improve clinical guidelines for detecting the pathogens of bacterial infectious pneumonia and VAP (4).

16S rDNA polymerase chain reaction (PCR)-denaturing gradient gel electrophoresis (DGGE) is one of the important methods used to investigate the diversity of microflora, and the gold standard for bacterial detection and classification is sequence analysis based on $16 \mathrm{~S}$ rDNA (5-7). Thus, the present study focused on examining the diversity of the microflora in the lower respiratory tract in order to elucidate the reasons neonates suffer from bacterial infectious pneumonia and VAP.

\section{Materials and methods}

Ethics statement. Prior to commencement of the present study, agreement was obtained from the Medical Ethics Committee of Chongqing Medical University (Chongqing, China), and the parents of the neonates provided signed informed consent prior to the experiment.

Patient selection. Newborns from the neonatal intensive care unit of the Children's Hospital of Chongqing Medical University between January 2012 and December 2012 were 
included in the present study. Newborn patients suffering from bacterial infectious pneumonia without VAP (IP group) and those suffering from bacterial infectious pneumonia combined with VAP (IVAP group) were included as the experimental groups, and those suffering from RDS without VAP (RDS group), were included as a positive control group. The negative control group comprised filter-sterilized double-distilled water for the extraction of DNA and subsequent PCR (7). There were 19 patients in the IP group, including 15 male and 4 female, and the gestational age was $37.1 \pm 3.3$ weeks. There were 8 patients in the IVAP group, including 7 male and 1 female, the gestational age was $30.3 \pm 3.9$ weeks.

The following exclusion criteria were used for RDS: Intrauterine infection, infectious diseases prior to intubation, and mothers with a history of infections or who had used antibiotics during the last month of pregnancy.

The following diagnostic criteria were used for VAP: Presence of rales or knock turbidity, with emerging purulent sputum, positive blood culture or epidemic strains isolated by endotracheal suction, and the presence of emerging pulmonary infiltrates, consolidation, cavities or pleural effusions, as indicated by X-ray examination (8-10).

Sample collection. Following 1, 3 and 5 days of ventilation, sputum samples were collected from the patients. The suction tube of the sputum culture collector was placed deep into the collection tube and, using negative pressure, 1-2 ml of sputum was aspirated. If the sputum thickness prohibited sputum collection, 1-3 $\mathrm{ml}$ of sterile saline was injected into the endotracheal tube, followed by five breathing cycles of the patients. Once the patient's oxygen saturation recovered, aspiration of sputum recommenced. All specimens were stored at $-20^{\circ} \mathrm{C}(11)$.

DNA extraction. The sputum samples were centrifuged at $4^{\circ} \mathrm{C}$ and $1,000 \mathrm{x} g$ for $1 \mathrm{~min}$, following which the supernatant was removed and the pellet was resuspended in $2 \mathrm{ml}$ of sterile saline. The sample was mixed and centrifuged again, in accordance with the above methods. Following two washes with sterile saline, the sample was analyzed using the Mini BEST Bacterial Genomic DNA Extraction kit (Ver2.0; Takara Biotechnology Co., Ltd., Dalian, China), in accordance with the manufacturer's protocol The negative control groups contained filter-sterilized double-distilled water for the extraction of DNA and PCR.

PCR amplification. The bacterial universal primers, designed according to the conserved $\mathrm{V} 3$ region of the bacterial $16 \mathrm{~S}$ rDNA gene were as follows: 357, forward 5'-CGCCCGGGG CGC GCCCCGGGCGGGGCGGGGGCAGGGGCCTAC GGGAGGCAGCAG-3' (including the $37 \mathrm{bp}$ 'GC' cap) and 518, reverse 5'-ATTACCGCGGCTGCTGG-3'. The amplifications were performed using an Eppendorf PCR machine (Eppendorf, Hamburg, Germany). The reaction volume was $50 \mu 1$ and included $6 \mu 1$ template DNA, $25 \mu 1$ Premix Taq Version 2.0 (Takara Bio, Inc., Otsu, Japan), $0.5 \mu l$ each primer and $18 \mu \mathrm{l}$ sterile $\mathrm{dd}_{2} \mathrm{O}$. The following reaction conditions were used: Initial denaturation at $94^{\circ} \mathrm{C}$ for $5 \mathrm{~min} ; 10$ cycles of denaturation at $94^{\circ} \mathrm{C}$ for $30 \mathrm{sec}$, annealing at $61-56^{\circ} \mathrm{C}\left(-0.5^{\circ} \mathrm{C} /\right.$ cycle $)$ and extension at $72^{\circ} \mathrm{C}$ for $1 \mathrm{~min} ; 25$ cycles of denaturation at $94^{\circ} \mathrm{C}$ for $30 \mathrm{sec}$, annealing at $56^{\circ} \mathrm{C}$ for $30 \mathrm{sec}$ and extension at $72^{\circ} \mathrm{C}$ for $1 \mathrm{~min}$; and a final extension at $72^{\circ} \mathrm{C}$ for $7 \mathrm{~min}$. A 2\% agarose gel, with 1X TAE (Tris base, acetic acid and EDTA) and 4S Green (Shanghai Biological Engineering Co., Ltd., Shanghai, China), was used to resolve the PCR products from the $5 \mu$ l. The target bands were $\sim 195 \mathrm{bp}$ in size. The remaining PCR products were stored at $-20^{\circ} \mathrm{C}$.

$D G G E$. A DCode system (Bio-Rad Laboratories, Inc., Hercules, CA, USA) was used to analyze the DGGE images. Each PCR product $(25 \mu \mathrm{l})$ was separated on an $8 \%$ polyacrylamide gel with a 35-65\% linear gradient of urea and formamide by electrophoresis at $85 \mathrm{~V}$ and $60^{\circ} \mathrm{C}$ for $16 \mathrm{~h}$. SYBR Green I (Tektronix Biotechnology Co., Ltd., Beijing, China) was used to stain the gel, and a Herolab UVT-20 M/W ultraviolet transilluminator was used to the image the gel. All the bands were excised, washed twice with $500 \mu 1$ sterile $\mathrm{ddH}_{2} \mathrm{O}$, mashed, placed in $30 \mu \mathrm{l}$ nuclease-free water and stored at $4^{\circ} \mathrm{C}$ overnight to elute the DNA. The supernatants were amplified with primers lacking the 'GC' cap under the same reaction conditions as described above. A $0.8 \%$ agarose gel was prepared with 1X TAE and 4S Green (Shanghai Biological Engineering Company). All the amplification products were electrophoresed at $110 \mathrm{~V}$ for $20 \mathrm{~min}$. An Agarose Gel DNA Purification $\mathrm{k}$ (version 2.0; Takara Bio, Inc.) was used to recover the DNA in the target bands and the DNA was stored at $-20^{\circ} \mathrm{C}(11)$.

Cloning and sequencing. A PMD18-T Vector system (Takara Bio, Inc.) was used to clone the PCR amplicons into a plasmid, and the Escherichia coli DH5 $\alpha$-competent cells (Tiangen, China) were transformed by the resulting clones. Luria Broth (LB) media containing ampicillin was used to culture the cells overnight at $37^{\circ} \mathrm{C}$, following which $1 \mathrm{ml}$ of this liquid was sent to Shanghai Biological Engineering Co., Ltd. for sequencing. The Basic Local Alignment Search Tool (blast.ncbi.nlm.nih.gov/Blast.cgi) was used to compare the results with the nucleotide databases in the National Center for Biotechnology Information GenBank (www.ncbi.nlm.nih.gov/).

Bacterial culture. The collected sputum oscillating fluid samples were inoculated onto Columbia blood agar plates and separate Haemophilus influenzae plates, and were cultured for $18-48 \mathrm{~h}$ at $37^{\circ} \mathrm{C}$. Gram staining was used to stain the resulting colonies, which were identified using a MicroScan WalkAway-40 (Siemens AG, Berlin, Germany) automated bacterial identification and susceptibility instrument.

Analysis of diversity and similarity. The numbers and the similarity of the bands from the DGGE images were measured using Quantity One version 4.62 (Bio Rad Laboratories, Inc.) software. The unweighted pair group method with arithmetic averages was used to analyze the cluster maps, and BIO-DAP version 2.0 software was used to calculate the Shannon-Wiener diversity index (Shannon-Wiener index) (11-13).

Statistical analysis. The data were analyzed using SPSS 17.0 statistical software (SPSS, Inc., Chicago, IL, USA). Data with a normal distribution are expressed as the mean \pm standard deviation. Comparisons between two groups were performed 
Table I. Clinical characteristics of patients in the IP and IVAP groups.

\begin{tabular}{|c|c|c|c|c|}
\hline Characteristic & IP $(n=19)$ & IVAP $(\mathrm{n}=8)$ & Statistical analysis & P-value \\
\hline Gender (male) & 15 & 7 & - & $1.000^{\mathrm{a}}$ \\
\hline Gestational age (weeks) ${ }^{\mathrm{c}}$ & $37.1 \pm 3.3$ & $30.3 \pm 3.9$ & $t=8.550$ & 0.007 \\
\hline Birth weight $(g)^{c}$ & $2,773 \pm 450$ & $1,876 \pm 667$ & $t=3.871$ & 0.060 \\
\hline Total intubation duration [P50 (P25-P75), days] & $4.25(2.90-5.10)$ & $14.70(8.10-17.70)$ & - & $0.000^{\mathrm{b}}$ \\
\hline Sputum culture results & & & - & $0.000^{\mathrm{a}}$ \\
\hline Negative (n) & 18 & 6 & - & - \\
\hline Normal flora (n) & 7 & 3 & - & - \\
\hline Klebsiella pneumoniae subspecies (n) & 4 & 9 & - & - \\
\hline Acinetobacter baumannii (n) & 0 & 3 & - & - \\
\hline Pseudomonas aeruginosa (n) & 0 & 2 & - & - \\
\hline Antibiotic & $\begin{array}{l}\text { Cefamandole/ } \\
\text { Cefoperazone, } \\
\text { sulbactam/ } \\
\text { Panipenem, } \\
\text { betamipron/ } \\
\text { Cefpiramide/ } \\
\text { Piperacillin, } \\
\text { tazobactam/ } \\
\text { Latamoxef }\end{array}$ & $\begin{array}{l}\text { Cefamandole/ } \\
\text { Cefoperazone, } \\
\text { sulbactam/ } \\
\text { Panipenem, } \\
\text { betamipron/ } \\
\text { Cefpiramide/ } \\
\text { Piperacillin, } \\
\text { tazobactam/ } \\
\text { Tienam/ } \\
\text { Metronidazole/ } \\
\text { Amphotericin/ } \\
\text { Fluconazole/ } \\
\text { Ciprofloxacin/ } \\
\text { Vancomycin }\end{array}$ & - & - \\
\hline Prognosis & & & - & $1.000^{\mathrm{a}}$ \\
\hline Improved and recovered (n) & 15 & 6 & - & - \\
\hline Succumbed to mortality (n) & 4 & 2 & - & - \\
\hline
\end{tabular}

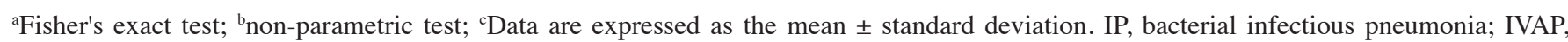
bacterial infectious pneumonia with ventilator-associated pneumonia.

using an independent sample $t$-test, and comparisons between several groups were performed using one-way analysis of variance or a pairwise least significant difference $t$-test when the variance was homogeneous. When the variance was heterogeneous, a non-parametric test was used. Data without a normal distribution are expressed as P50 (P25-P75), and were analyzed using the non-parametric test method. Count data were analyzed using a $\chi^{2}$ test when $\mathrm{n} \geq 40$ and $\mathrm{T} \geq 1$, and the extract method was used when $\mathrm{n}<40$ or $\mathrm{T}<1$. $\mathrm{P}<0.05$ was considered to indicate a statistically significant difference. The pairwise comparisons of multiple count data were performed when the test level was fixed.

\section{Results}

Clinical characteristics. A total of 42 newborn patients were suitable for participation in the present study due to meeting the inclusion criteria, which included 15 patients in the RDS group, 19 in the IP group and eight in the IVAP group. The clinical data for the experimental groups (IP group and IVAP group) are listed in Table I. Cefoxitin was used empirically in the
Table II. Groupings based on the DNA amplification of 73 sputum samples.

\begin{tabular}{lccr}
\hline Day & RDS & IP & IVAP \\
\hline 1 & 10 & 15 & 7 \\
3 & 7 & 12 & 6 \\
5 & 5 & 6 & 5 \\
Total & 22 & 33 & 18 \\
\hline RDS, respiratory & distress syndrome; IP, & bacterial infec- \\
tious pneumonia; IVAP, bacterial infectious & pneumonia with \\
ventilator-associated pneumonia. & & \\
\hline
\end{tabular}

patients in the RDS group. The patients in of the IP and IVAP groups with positive culture results were treated according to the susceptibility data, and those with negative culture results were treated according to empirical treatments. The patients in the IVAP group were considered to be VAP within 1.9-6.5 days 


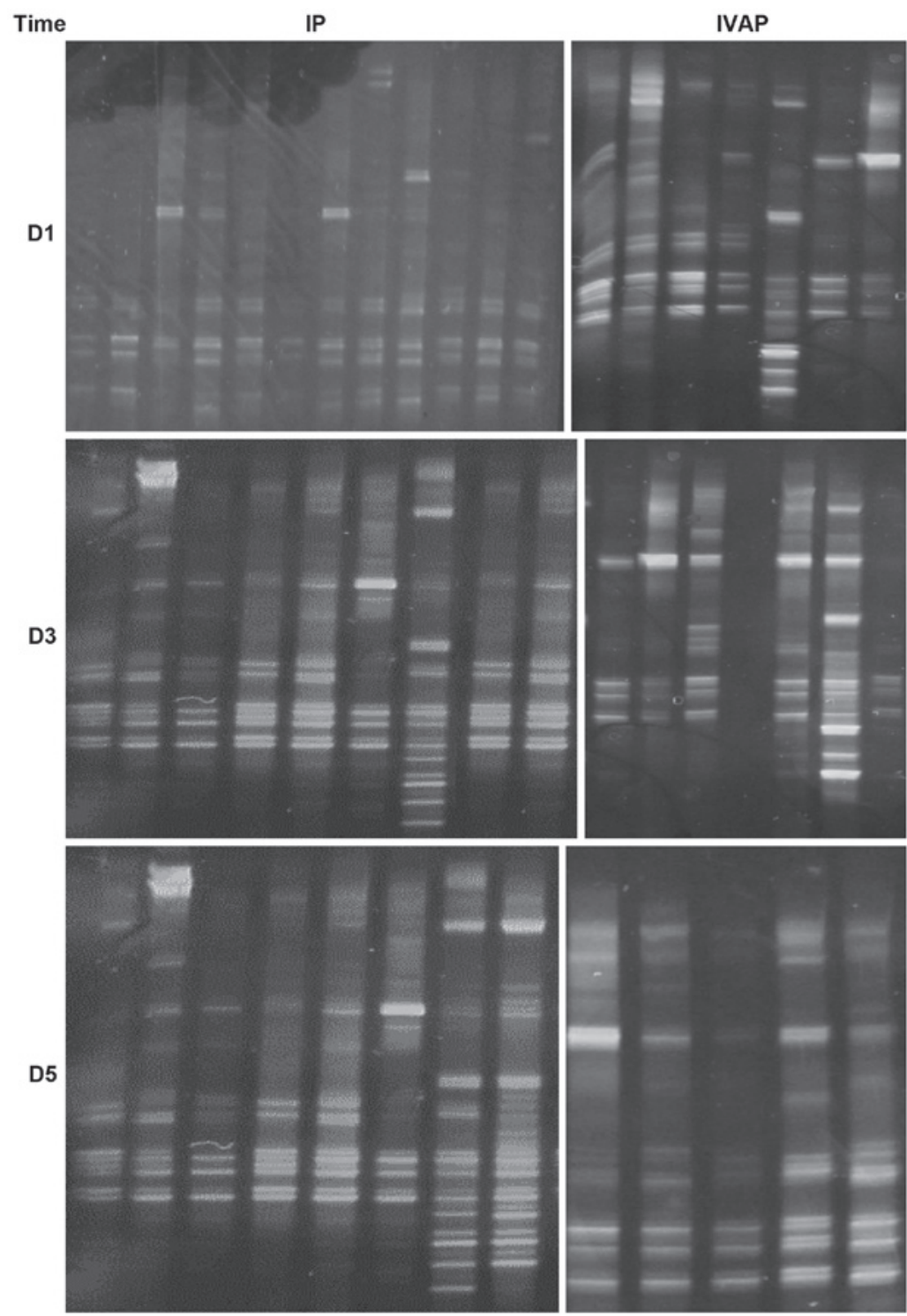

Figure 1. Denaturing gradient gel electrophoresis images of the amplified DNA products in the patient groups. IP, bacterial infectious pneumonia; IVAP, bacterial infectious pneumonia with ventilator-associated pneumonia; D, day.

(P50=3.2) following intubation. The gestational age was lower and the intubation duration was longer for the patients in the IVAP group, compared with those in the IP group.

A total of 73 sputum samples were sent for culture in the Children's Hospital of Chongqing Medical University, and the detection ratio was $54.4 \%$. The detection ratio for the IP group was lower, compared with for the IVAP group.

Sample collection, DNA extraction and PCR. A total of 73 samples were collected. The target bands ( 195 base pairs) were obtained from 73 sputum samples $(82.0 \%)$, which are presented in Table II. No target band was generated from the negative control group samples.

$D G G E$ images. Visible bands were found in all 73 sputum samples, as shown in Fig. 1.

Sequencing results. A total of 11 species were detected following the isolation, cloning and sequencing of the DGGE bands (Table III).
Analysis of diversity. The bacterial diversity of a sample is determined by the number of bands in a lane; if the former is high, the latter is high (14). In the present study, the number of bands in each group were determined, as shown in Fig. 2. As indicated by the results, with prolonged intubation, alterations in the diversity was observed in each group, as follows: The diversity of the RDS group initially increased and then leveled off, whereas the IP group showed a gradual increase in diversity. The IVAP group initially decreased, followed by an increase. Comparison between the groups within the same time period indicated no differences in diversity within the first day of intubation, and the levels of diversity were in the order of RDS group > IP group > IVAP group on days 1-5 post-intubation. The data of the Shannon-Wiener index, shown in Fig. 3, showed that the overall trends and associations were consistent with the above levels of diversity.

Similarity analysis of microflora. As shown in Fig. 4, the similarity results of each group were examined. With prolonged intubation, there was no significant change in the similarity 
Table III. Sequencing results of the bands in the denaturing gradient gel electrophoresis images.

\begin{tabular}{llr}
\hline NCBI BLAST result & Accession number & Identity (\%) \\
\hline Serratia sp. & KC182731.1 & 100 \\
Achromobacter sp. & HE613447.1 & 100 \\
Klebsiella sp. & KC354804.1 & 100 \\
Staphylococcus sp. & JX849039.1 & 100 \\
Acinetobacter sp. & KC245151.1 & 99 \\
Streptococcus sp. & JX861486.1 & 100 \\
Pseudomonas sp. & KC415769.1 & 100 \\
Macrococcus sp. & HQ238716.1 & 100 \\
Brevundimonas sp. & JX950099.1 & 99 \\
Actinomyces sp. & HM854563.1 & 99 \\
Schlegel sp. & AY538706.1 & 99 \\
\hline
\end{tabular}

NCBI, National Center for Biotechnology Information; BLAST, Basic Local Alignment Search Tool.

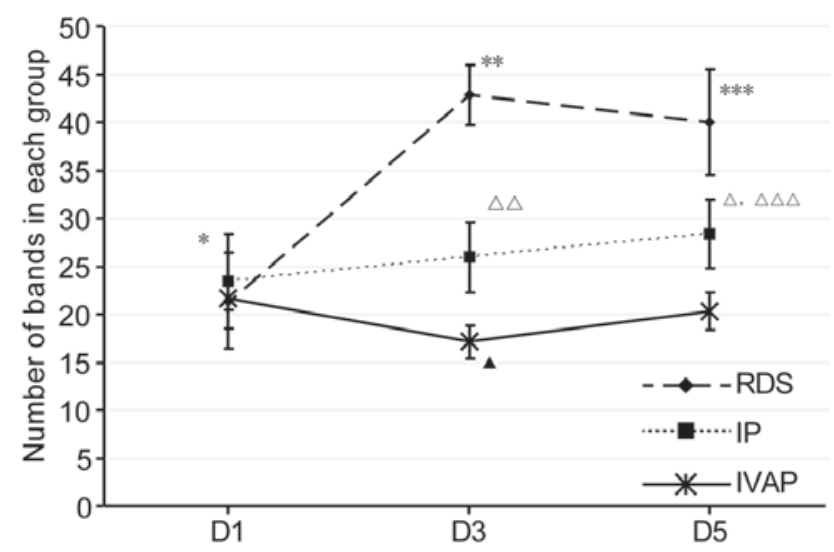

Figure 2. Number of bands in each group. Data are expressed as the mean \pm standard deviation. RDS, respiratory distress syndrome; IP, bacterial infectious pneumonia; IVAP, bacterial infectious pneumonia with ventilator-associated pneumonia; D, day. ${ }^{*} \mathrm{P}<0.05$, RDS D1 vs. RDS D3 and RDS D5 groups; ${ }^{* *} \mathrm{P}<0.05$, RDS D3 vs. IP D3 and IVAP D3 groups; ${ }^{* * *} \mathrm{P}<0.05$, RDS D5 vs. IP D5 and IVAP D5 groups; ${ }^{\triangle} \mathrm{P}<0.05$, IP D5 vs. IP D1 and IP D5 groups; $\triangle \triangle \mathrm{P}<0.05$, IP D3 vs. IVAP D3 group; ${ }^{\triangle} \triangle \mathrm{P}<0.05$, IP D5 vs. IVAP D5 group, ${ }^{\mathbf{}} \mathrm{P}<0.05$, IVAP D3 vs. IVAP D1 and IVAP D5 groups.

of the RDS group in the lower respiratory tract, whereas the similarity levels of the other two groups were maintained at an initial increased level. Comparison between the groups within the same time period revealed no differences in similarity within the first day of intubation, whereas the similarity levels on days 1-5 post-intubation were in the order, IVAP group $>$ IP group $>$ RDS group.

Composition of the microflora. The variety of the genera in each group and their composition ratios are shown in Table IV and Fig. 5.

In total, 11 bacterial genera were detected, and dynamic alterations were observed in the composition and constituent ratios of the bacterial genera in the three groups with prolonged intubation. In the three groups, six genera were detected:

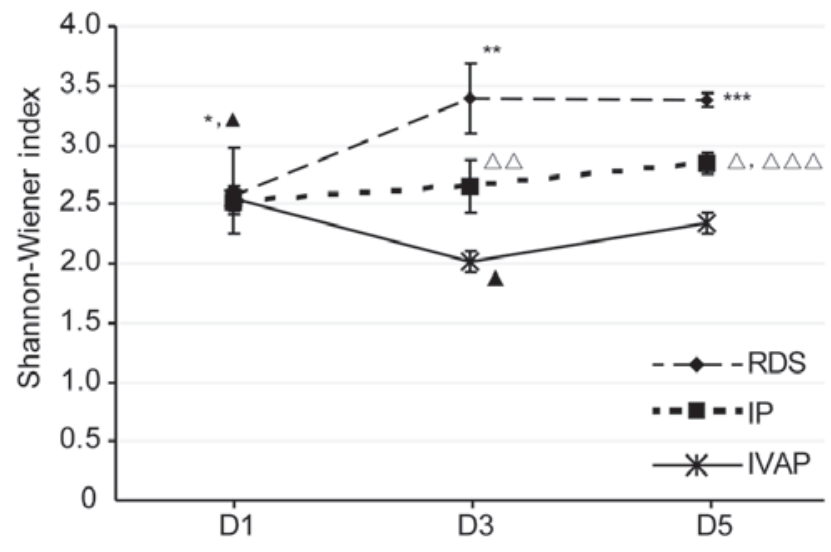

Figure 3. Shannon-Wiener Index for each group. Data are expressed as the mean \pm standard deviation. RDS, respiratory distress syndrome; IP, bacterial infectious pneumonia; IVAP, bacterial infectious pneumonia with ventilator-associated pneumonia; D, day. ${ }^{*} \mathrm{P}<0.05$, RDS D1 vs. RDS D3 and RDS D5 groups; ${ }^{* *} \mathrm{P}<0.05$, RDS D3 vs. IP D3 and IVAP D3 groups; ${ }^{* * *} \mathrm{P}<0.05$, RDS D5 vs. IP D5 and IVAP D5 groups; $\triangle \mathrm{P}<0.05$, IP D5 vs. IP D1 and IP D5 groups; ${ }^{\triangle} \triangle \mathrm{P}<0.05$, IP D3 vs. IVAP D3 group; ${ }^{\triangle} \triangle \mathrm{P}<0.05$, IP D5 vs. IVAP D5 group, ${ }^{\mathbf{\Delta}} \mathrm{P}<0.05$, IVAP D1 vs. IVAP D3 and IVAP D5 groups; ${ }^{\wedge} \mathrm{P}<0.05$, IVAP D3 vs. IVAP D5 group.

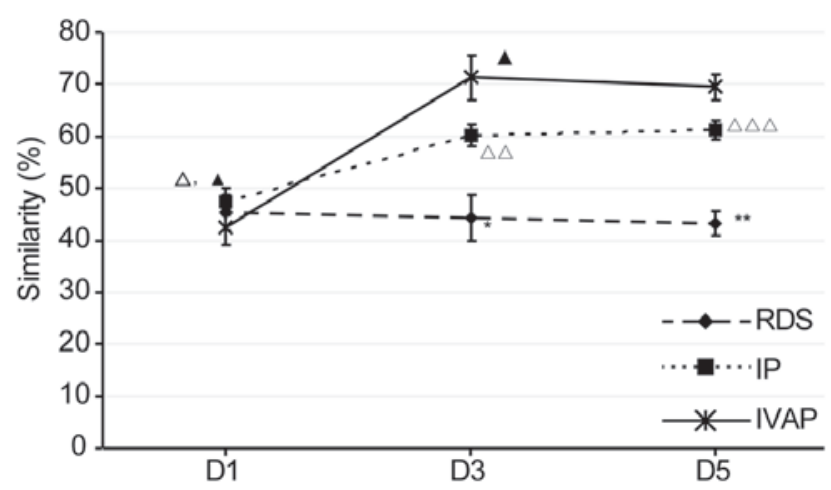

Figure 4. Similarity of the sputum in each group. Data are expressed as the mean \pm standard deviation. RDS, respiratory distress syndrome; IP, bacterial infectious pneumonia; IVAP, bacterial infectious pneumonia with ventilator-associated pneumonia; D, day. ${ }^{*} \mathrm{P}<0.05$, RDS D3 vs. IP D3 and IVAP D3 groups; ${ }^{* *} \mathrm{P}<0.05$, RDS D5 vs. IP D5 and IVAP D5 groups; ${ }^{\triangle} \mathrm{P}<0.05$, IP D1 vs. IVAP D3 group; ${ }^{\triangle} \triangle \mathrm{P}<0.05$, IP D3 vs. IVAP D3 group; $\triangle \triangle \triangle \mathrm{P}<0.05$, IP D5 vs. IVAP D5 group, ${ }^{\wedge} \mathrm{P}<0.05$, IVAP D1 vs. IVAP D3 and IVAP D5 groups.

Klebsiella sp., Serratia sp., Streptococcus sp., Achromobacter sp., Acinetobacter sp. and Staphylococcus sp. Staphylococcus sp. on the first day of intubation. With prolonged intubation, the composition ratio of Streptococcus sp. gradually increased, whereas Serratia sp. exhibited a trend of gradual reduction. Pseudomonas sp. was not present in the RDS group.

The composition ratios of the bacterial genera in the three groups were compared following the same duration of intubation No differences were observed between the IP group and IVAP group on the first day of intubation. The composition ratios of Serratia sp. and Acinetobacter sp. in the RDS group were higher, compared with those in the IP group and IVAP group, whereas the composition ratios of Klebsiella sp., Achromobacter sp. and Streptococcus sp. in the RDS group were lower, compared with those in the other two groups. The IVAP group showed the highest composition ratios of Klebsiella sp. and Streptococcus sp. on days 1-3 post-intubation, followed by the IP group and the 
Table IV. Composition ratios of the genera in each group.

\begin{tabular}{|c|c|c|c|c|c|c|c|c|c|}
\hline \multirow[b]{2}{*}{ Genus } & \multicolumn{3}{|c|}{$\mathrm{RDS}$} & \multicolumn{3}{|c|}{ IP } & \multicolumn{3}{|c|}{ IVAP } \\
\hline & D1 & D3 & D5 & D1 & D3 & D5 & D1 & D3 & D5 \\
\hline Serratia $(\%)$ & 5.5 & 19.5 & 27.9 & 14.8 & 25.4 & 27.0 & 16.5 & 35.3 & 15.4 \\
\hline Achromobacter (\%) & 69.1 & 57.2 & 39.5 & 52.9 & 47.8 & 37.9 & 54.2 & 42.4 & 28.5 \\
\hline Klebsiella $(\%)$ & 9.1 & 9.7 & 14.0 & 6.6 & 8.0 & 11.8 & 5.2 & 6.1 & 8.7 \\
\hline Staphylococcus (\%) & 2.0 & 3.4 & 9.3 & 5.3 & 7.9 & 14.4 & 4.7 & 10.0 & 19.1 \\
\hline Acinetobacter $(\%)$ & 9.1 & 10.3 & 9.3 & 2.1 & 3.4 & 7.8 & 3.2 & 4.1 & 6.6 \\
\hline Streptococcus (\%) & 0 & 0 & 0 & 0.3 & 1.9 & 0 & 0.5 & 0 & 3.1 \\
\hline Pseudomonas (\%) & 5.3 & 0 & 0.0 & 17.9 & 0 & 0 & 15.7 & 0 & 0 \\
\hline Macrococcus (\%) & 0 & 0 & 0 & 0 & 2.5 & 0 & 0 & 0 & 5.8 \\
\hline Brevundimonas (\%) & 0 & 0 & 0 & 0 & 1.5 & 0 & 0 & 0 & 7.3 \\
\hline Actinomyces (\%) & 0 & 0 & 0 & 0 & 1.7 & 0 & 0 & 0 & 5.7 \\
\hline Schlegel (\%) & 0 & 0 & 0 & 0 & 0 & 1.1 & 0 & 2.1 & 0 \\
\hline$\chi^{2}$ & & 38.230 & & & 32.661 & & & 43.623 & \\
\hline P-value & & 0.000 & & & 0.000 & & & 0.000 & \\
\hline
\end{tabular}

RDS, respiratory distress syndrome; IP, bacterial infectious pneumonia; IVAP, bacterial infectious pneumonia with ventilator-associated pneumonia; D, day.

$\square$ Schlegel sp.

$\square$ Actinomyces sp.

$\square$ Brevundimonas $s p$.
- Macrococcus sp.

$\square$ Staphylococcus sp.

- Pseudomonas sp.

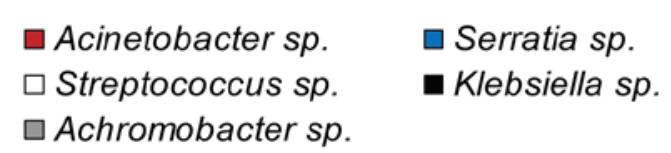

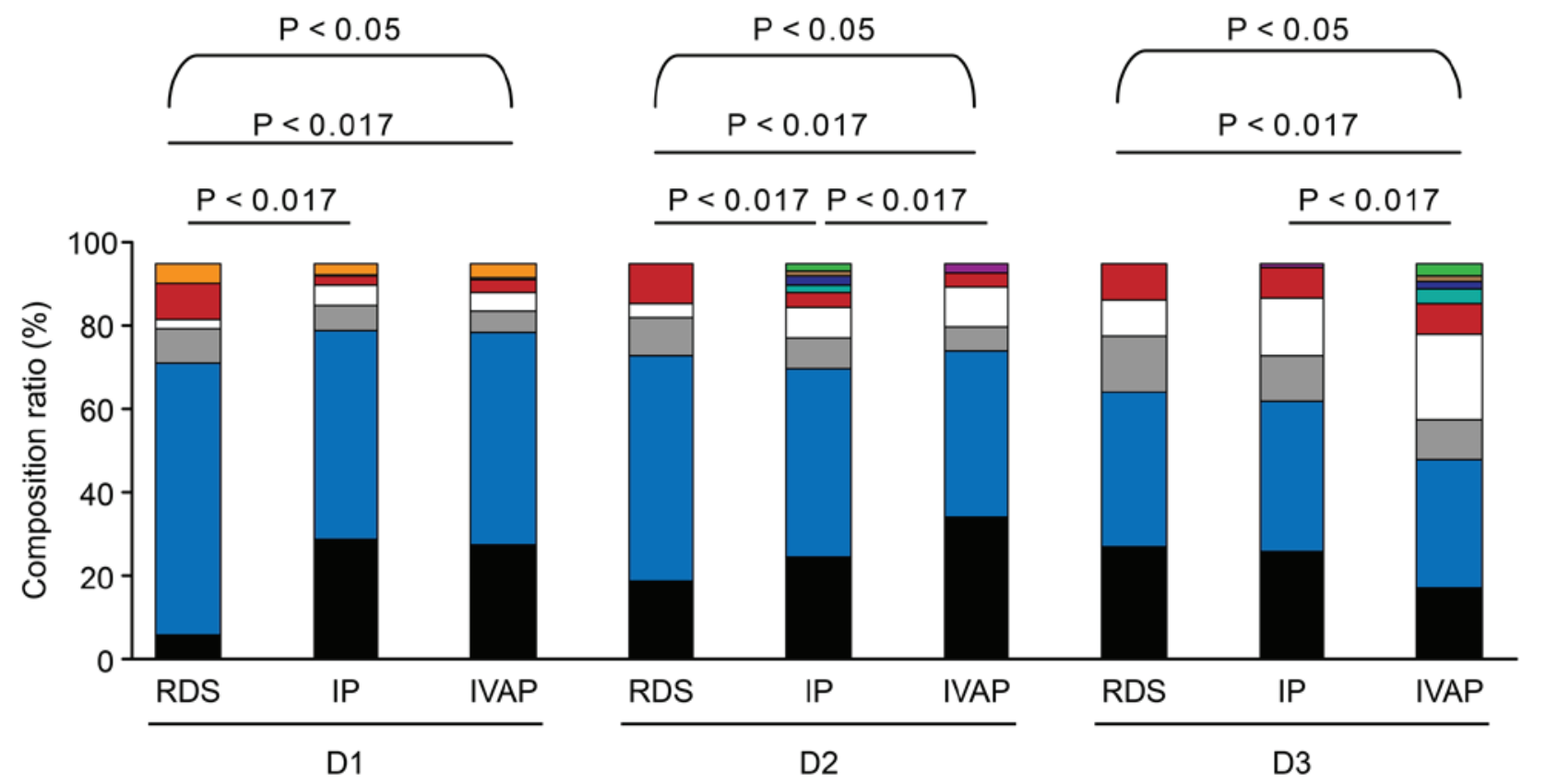

Figure 5. Composition ratios of the genera in each group. The fixed test level was 0.017 when pairwise comparisons of multiple count data were performed. RDS, respiratory distress syndrome; IP, bacterial infectious pneumonia; IVAP, bacterial infectious pneumonia with ventilator-associated pneumonia; D, day.

RDS group, which exhibited the lowest ratio. The composition ratios of Serratia sp. and Achromobacter sp. were in the order IVAP group < IP group < RDS group, whereas the constituent ratio of Acinetobacter sp. was in the order IP group < IVAP group < RDS group. On days 3-5 post-intubation, no differences were observed between the RDS group and IP group, however, the IVAP group showed lower constituent ratios of Serratia sp., Achromobacter sp., Acinetobacter sp. and Klebsiella sp., and a higher composition ratio of Streptococcus sp., compared with the RDS group and IP group. 
Comparison of the detection rate between the two methods. In the present study, the detection rate determined using the $16 \mathrm{~S}$ rDNA PCR-DGGE cloning-sequencing method was $82.0 \%$ (73/89), whereas the rate determined using the culture method was $54.4 \%\left(\chi^{2}=17.092 ; \mathrm{P}<0.001\right)$, which showed that the former method had higher efficiency, compared with the latter method.

\section{Discussion}

Associations between the microfloral diversity of the respiratory tract and neonatal infectious pneumonia with concurrent $V A P$. No differences were observed in the microfloral diversity among the RDS, IP and IVAP groups in the first day of intubation. This was likely due to the low number of bacteria entering the lower respiratory tract through the throat and endotracheal tubing in the early stages of intubation in the patients with RDS, resulting in a low level of diversity. The reduced diversity of the respiratory microflora may also have been due to infection in the patients with IP and IVAP. In addition, no VAP complications were present in the patients with IVAP within the first day of intubation; thus, the microfloral diversity in the patients with IVAP was equivalent to that in the patients with IP. Therefore, microfloral diversity was found to decrease in the RDS, IP and IVAP groups in the first day of intubation, with no difference in diversity observed among the three groups.

On days 1-3 post-intubation, the RDS group showed the highest level of microfloral diversity, followed by the IP group and the IVAP group, which had the lowest level of diversity. The patients with IVAP had the lowest microfloral diversity as they were afflicted with more severe infections, namely VAP, in addition to their preceding pneumonia. Only one type of pneumonia was present in the patients with IP, who showed an intermediate level of respiratory microfloral diversity (7). These findings indicated that more severe pneumonia was associated with reduced microfloral diversity.

The microfloral diversity among the three groups at 3-5 days post-intubation was comparable with the diversity at 1-3 days post-intubation. The microfloral diversity of the IP group increased, but remained lower than that of the RDS group, indicating more severe infection and poorer overall prognoses in the patients of the IP group. Thus, patients with IP may require additional time to recover completely. The diversity of the IVAP group remained the lowest, which was likely to be associated with the highest severity of infection and the inhibitory effects of antibiotics.

In the present study, the microfloral similarity was highest in the IVAP group, followed by the IP group and RDS group. This finding indicated that a higher severity of infection caused higher levels of microfloral similarity and more marked inhibitory effects on microfloral diversity (14).

Association between the composition of respiratory microflora and pneumonia. A total of 11 bacterial genera were detected in the lower respiratory tract, and the microfloral constituent ratio exhibited common features among the three groups. One common feature was the dynamic changes observed in the number, type and constituent ratios of the bacterial genera in the three groups following prolonged intubation, and another common feature was that six genera were shared by the three groups.

Compared with the RDS group, microfloral imbalance was observed in the IP and IVAP groups, manifested as follows: i) Changes in the composition of common bacterial genera. Within 1 day post-intubation, increased ratios of Klebsiella sp., Streptococcus sp. and Achromobacter sp., and reduced ratios of Serratia sp. and Acinetobacter sp. suggested possible infectious pneumonia. On days 1-3 post-intubation, the composition of Klebsiella sp. and Streptococcus sp. were the highest in the IVAP group, followed by the IP group and RDS group; however, the composition of Serratia sp., Achromobacter sp. and Acinetobacter sp. were lowest in the IVAP group, higher in the IP group and highest in the RDS group. Therefore, the increased composition of Klebsiella sp. and Streptococcus sp., together with the reduced ratios of Serratia sp. and Acinetobacter sp. suggested possible VAP complications in the patients with IP. At 3-5 days post-intubation, no differences in the constituent ratios were observed between the RDS group and IP group, which was likely to be due to certain patients with IP showing improvement in their condition and certain RDS patients showing concurrent infection. In addition, the IVAP group exhibited lower constituent ratios of Serratia sp., Achromobacter sp., Acinetobacter sp., Klebsiella sp. and Streptococcus sp., compared with those of the RDS and IP groups. This observation was likely associated with the suppression from antibiotics, disease outcome and other types of bacteria present in the IVAP group. 2) Pseudomonas sp., Brevundimonas sp., Actinomyces sp. and other genera were detected in the IP and IVAP groups, indicating changes in bacterial composition with infectious pneumonia, the suppression of dominant bacteria and an increase of opportunistic pathogenic bacteria $(15,16)$.

In the present study, Klebsiella subspecies and Pseudomonas aeruginosa were detected in the bacterial culture. In addition, the compositions of Klebsiella sp., to which Klebsiella subspecies belong, were higher in the IP and IVAP groups, compared with the RDS group. Pseudomonas sp., to which Pseudomonas aeruginosa belongs, was not detected in the RDS group. This suggested that, when a bacterial strain is present in the culture and the constituent ratio of its bacterial genus is higher, compared with that of the control group, or the bacterial genus is newly detected, this bacterial strain is likely a pathogenic bacterial strain. However, although Acinetobacter baumannii was also detected in the culture in the present study, the constituent ratio of its bacterial genus, Acinetobacter sp., was lower in the IP and IVAP groups, compared with the RDS group. This finding was inconsistent with the above results. Acinetobacter baumannii is a multi-drug resistant opportunistic pathogen, and it is frequently found in the lower respiratory tract of patients with severe pneumonia, Guillain-Barre syndrome or traumatic brain injury who are supported with mechanical ventilation $(17,18)$. The majority of the patients exhibited improvements in their condition with continued treatment of the originally prescribed antibiotics, or even without the administration of antibiotics, which indicated that the Acinetobacter baumannii present in the culture only colonized the respiratory tract and was not pathogenic. This suggested that, when a bacterial strain 
is present in culture and the constituent ratio of its bacterial genus is higher, compared with that of the control group, the bacterial strain is most likely not pathogenic. This conclusion requires comprehensive analysis based on clinical manifestations and other laboratory examination, however the analysis of changes in constituent ratios of bacterial genera in the lower respiratory tract can assist in determining the condition of pneumonia and whether a bacteria strain is pathogenic.

Comparison between the $16 S$ rDNA-PCR-DGGE cloning-se quencing method and culture method. In the present study, 11 bacterial genera were detected in sputum samples using the $16 \mathrm{~S}$ rDNA-DGGE cloning-sequencing method. The three bacterial strains detected in the sputum culture, Klebsiella pneumoniae subspecies, Acinetobacter baumannii and Pseudomonas aeruginosa, belong to Klebsiella sp., Acinetobacter sp. and Pseudomonas sp., respectively, indicating consistent results using the two methods.

Among the 52 samples collected from the patients with IP and IVAP and used for detection in sputum culture, 24 samples did not show either bacterial growth or normal microfloral growth. The bacterial genera detected using the sequencing method, including Serratia sp., Achromobacter sp., Streptococcus sp., Staphylococcus sp., Actinomyces sp., Brevundimonas sp., Macrococcus sp. and Schlegel sp., were not detected using the sputum culture method, indicating that the 16S rDNA-DGGE cloning-sequencing method was more sensitive and detected bacteria, which were not detected through bacterial culture. These results showed that the detection efficiency of the $16 \mathrm{~S}$ rDNA-DGGE cloning-sequencing method was higher, compared with that of the culture method. Therefore, the 16S rDNA-DGGE cloning-sequencing method was considered to be suitable for use as a supplement to the culture method.

In conclusion, the present study demonstrated that: i) Microfloral imbalances in the lower respiratory tract of newborns with bacterial pneumonia caused a reduction in microfloral diversity, and the increased severity of infection was associated with the lower diversity. The microfloral diversity was ordered as follows: IVAP group $<$ IP group $<$ RDS group. ii) Reductions in microfloral diversity were found in the lower respiratory tract of newborns with bacterial pneumonia. Increased constituent ratios of Klebsiella sp. and Streptococcus sp., and reduced constituent ratios of Serratia sp. and Acinetobacter sp. provided an early indicator of the occurrence of VAP.

\section{Acknowledgements}

The present study was supported by the National Natural Science Foundation of China (grant no. 81370744), the Doctoral Degree Funding from the Chinese Ministry of Education (grant no. 20135503110009), the State Key Clinic Discipline Project (grant no. 2011-873) and the subproject of the National Science \& Technology Pillar Program during the Twelfth Five-year Plan Period in China (grant no. 2012BAI04B05).

\section{References}

1. Peters BM, Jabra-Rizk MA, O'May GA, Costerton JW and Shirtliff ME: Polymicrobial interactions: Impact on pathogenesis and human disease. Clin Microbiol Rev 25: 193-213, 2012.

2. Balter M: Taking stock of the human microbiome and disease. Science 336: 1246-1247, 2012.

3. Yalaz M, Altun-Köroğlu O, Ulusoy B, Yildiz B, Akisu M, Vardar F, Ozinel MA and Kültürsay N: Evaluation of device-associated infections in a neonatal intensive care unit. Turk J Pediatr 54: 128-135, 2012.

4. Kellenberger E: Exploring the unknown. The silent revolution of microbiology. EMBO Rep 2: 5-7, 2001.

5. Relman DA, Loutit JS, Schmidt TM, Falkow S and Tompkins LS: The agent of bacillary angiomatosis. An approach to the identification of uncultured pathogens. N Engl J Med 323: 1573-1580, 1990.

6. Relman DA, Schmidt TM, MacDermott RP and Falkow S: Identification of the uncultured bacillus of Whipple's disease. N Engl J Med 327: 293-301, 1992.

7. Cairns S, Thomas JG, Hooper SJ, Wise MP, Frost PJ, Wilson MJ, Lewis MA and Williams DW: Molecular analysis of microbial communities in endotracheal tube biofilms. PLoS One 6: e14759, 2011.

8. Grgurich PE, Hudcova J, Lei Y, Sarwar A and Craven DE: Diagnosis of ventilator-associated pneumonia: Controversies and working toward a gold standard. Curr Opin Infect Dis 26: 140-150, 2013.

9. Craven DE, Hudcova J and Lei Y: Diagnosis of ventilator-associated respiratory infections (VARI): Microbiologic clues for tracheobronchitis (VAT) and pneumonia (VAP). Clin Chest Med 32: 547-557, 2011.

10. Wang $\mathrm{F}$ and $\mathrm{He} \mathrm{B}$ : The role of endotracheal aspirate culture in the diagnosis of ventilator-associated pneumonia: A meta analysis. Zhonghua Jie He He Hu Xi Za Zhi 36: 27-32, 2013 (In Chinese).

11. Payne MS, Goss KC, Connett GJ, Kollamparambil T, Legg JP, Thwaites R, Ashton M, Puddy V, Peacock JL and Bruce KD: Molecular microbiological characterization of preterm neonates at risk of bronchopulmonary dysplasia. Pediatr Res 67: 412-418, 2010.

12. Wang Y, Hoenig JD, Malin KJ, Qamar S, Petrof EO, Sun J, Antonopoulos DA, Chang EB and Claud EC: 16S rRNA gene-based analysis of fecal microbiota from preterm infants with and without necrotizing enterocolitis. ISME J 3: 944-954, 2009.

13. Hill TC, Walsh KA, Harris JA and Moffett BF: Using ecological diversity measures with bacterial communities. FEMS Microbiol Ecol 43: 1-11, 2003.

14. Signoretto C, Bianchi F, Burlacchini G, Sivieri F, Spratt D and Canepari P: Drinking habits are associated with changes in the dental plaque microbial community. J Clin Microbiol 48: 347-356, 2010.

15. Codling C, O'Mahony L, Shanahan F, Quigley EM and Marchesi JR: A molecular analysis of fecal and mucosal bacterial communities in irritable bowel syndrome. Dig Dis Sci 55: 347-356, 2010

16. Noor SO, Ridgway K, Scovell L, Kemsley EK, Lund EK, Jamieson C, Johnson IT and Narbad A: Ulcerative colitis and irritable bowel patients exhibit distinct abnormalities of the gut microbiota. BMC Gastroenterol 10: 134, 2010.

17. Song W, Wu YM, Ji Z, Zhu JJ and Pan SY: Guillain-Barré syndrome following sepsis after stereotactic aspiration for spontaneous pontine hemorrhage. Neurol Sci 3: 657-660, 2012.

18. Reddy D, Morrow BM and Argent AC: Acinetobacter baumannii infections in a South African paediatric intensive care unit. J Trop Pediatr 3: 182-187, 2015. 